\title{
Sex lethal and upstream ORFs: a bait-and-trap system for ribosomes
}

\author{
Peng Yao and Paul L Fox*
}

\begin{abstract}
An upstream ORF cooperates with Sex lethal to inhibit msl-2 translation during Drosophila sex determination, a biological principle with potentially broad application in gene regulation.
\end{abstract}

Upstream open reading frames (uORFs) in mRNAs are short sequences in the 5 ' untranslated region (UTR) characterized by a start codon, a short open reading frame (ORF) and a stop codon, all upstream of the major start codon that initiates translation of the principal gene product. (The term UTR is actually a misnomer, precisely because of the existence of uORFs, and is more accurately referred to as transcript leader region [1].) A ribosome encountering a uORF has several options, including scanning through the uORF until the major ORF is encountered, translating the $\mathrm{UORF}$ and then reinitiating translation of the major ORF, or translating the uORF and then stalling. The latter case can lead to decreased translation of the major ORF, or possibly shortened mRNA half-life by nonsense-mediated decay [1]. Thus, uORFs present diverse opportunities for regulation of gene expression. The likely importance of uORFs is suggested by their presence in about half of all annotated transcripts in both humans and mice [2]. Analysis of a group of uORF-containing mRNAs showed that uORFs reduce protein expression by 30 to $80 \%$. uORF-altering mutations have been predicted or confirmed in more than a dozen human-disease-associated genes [2]. The molecular mechanisms underlying the effect of uORFs on translation are not well understood. There is overwhelming evidence for the importance of mRNA secondary structure in uORF-mediated regulation of translation [3,4]; however, a role for specific 5' UTR-binding proteins is less certain. In an elegant report in Cell, Matthias

*Correspondence: foxp@ccf.org

Department of Cell Biology, The Lerner Research Institute, Cleveland Clinic, Cleveland, OH 44195, USA
Hentze and colleagues [5] elucidate a uORF-dependent mechanism by which the Drosophila mRNA-binding protein Sex lethal (Sxl) inhibits translation of its target transcript, male-specific lethal (msl)-2.

\section{Sex determination by dual interaction of SxI with msl-2 mRNA}

In Drosophila, dosage compensation equalizes the expression of X-linked genes in males and females. In male flies, the $m s l$-2-containing dosage compensation complex is required for hypertranscription of the single $\mathrm{X}$ chromosome [6]. In female flies, msl-2 mRNA is translationally repressed by interaction of Sxl with poly(U) stretches in both the 5' and the 3' UTRs [7]. This dual-action, sequential blocking mechanism comprises a fail-safe system that completely silences msl-2 mRNA translation. The molecular mechanism underlying repression of $m s l-2$ mRNA translation by Sxl interaction with the 3' UTR has been established [8]. Sxl, together with a co-repressor protein, Upstream of N-Ras (UNR), binds a poly(U) stretch in the msl-2 mRNA 3' UTR and inhibits recruitment of the 43S preinitiation complex (PIC) to the mRNA 5' terminus (Figure 1). However, the mechanism underlying Sxl-regulated, 5' UTR-mediated translational repression of the $m s l-2$ mRNA until now has remained a mystery.

\section{The role of a uORF in inhibition of $m s /-2$ translation by SxI}

Taking advantage of a cell-free translation system derived from Drosophila embryos, the recent publication from the Hentze laboratory [5] reveals that a uORF synergizes with Sxl to inhibit $m s l-2$ mRNA translation. They show that $m s l-2$ mRNA contains three uORFs upstream of the Sxl binding site, but only the 3'-most of the uORFs is essential for Sxl-directed translational control. Mutation analysis of the uORF and its immediate surroundings indicated that recognition of the UAUG upstream initiation site by scanning ribosomes is essential for control; however, regulation is independent of the coding sequence of the UORF and of its elongation and termination. As expected, both Sxl and the poly(U) binding site in the 5' UTR are also essential for uORF-mediated translational control. Interestingly, the critical sequence elements are 


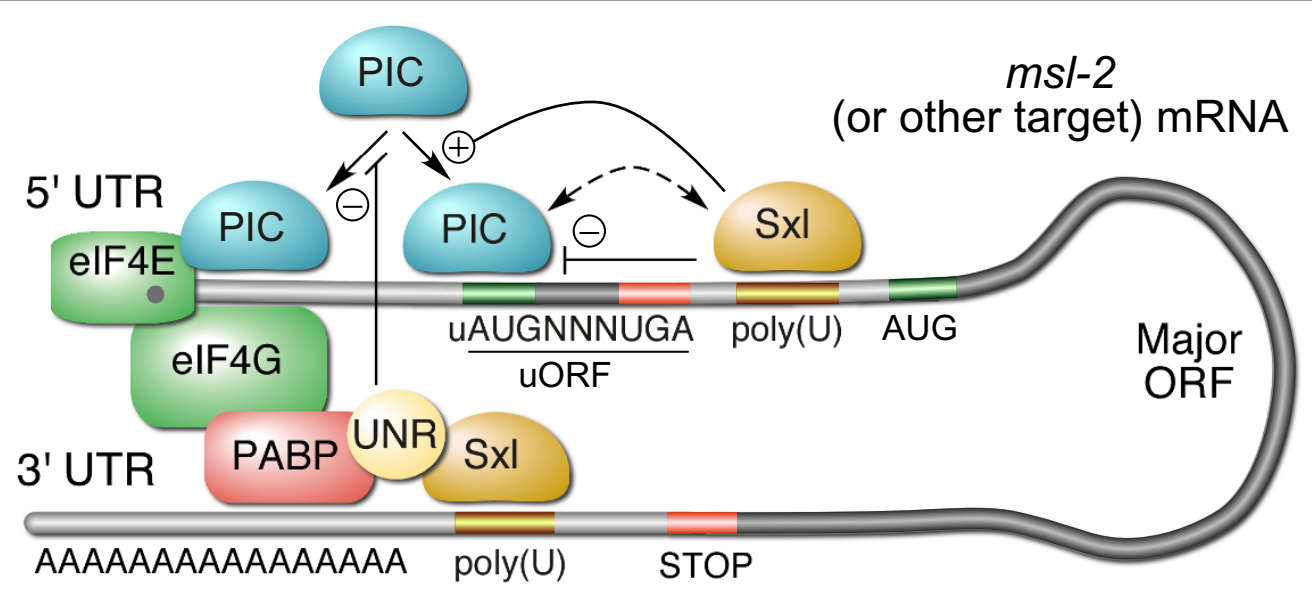

Figure 1. The translational control pathway of $m s l-2$ mRNA in sex determination in Drosophila. The main features of the gene are shown, including the upstream ORF (UORF) with its consensus sequence, two poly(U) stretches, the translation start and stop codons and the poly(A) tail, together with the proteins involved in this regulation. elF, eukaryotic translation initiation factor; $m s /-2$, male-specific lethal-2; PABP, poly(A) binding protein; PIC, 43 S preinitiation complex; SxI, Sex lethal; UNR, co-repressor protein Upstream of N-Ras; UTR, untranslated region. Positive and negative influences of the binding of $S x \mid$ to poly(U) stretches in $5^{\prime}$ and $3^{\prime} U T R s$ are indicated by plus and minus signs, respectively. The potential interaction between Sxl and PIC is indicated by a dashed double-headed arrow.

well-conserved in a dozen Drosophilid species, notably the upstream optimal context, the presence of precisely one codon between the UAUG and UGA, and a downstream poly(U) stretch separated from the uORF by a spacer of variable sequence and length. The role of the proximal pair of uORFs in the context of translational regulation by Sxl was clearly ruled out by deletion analysis [5], but it is interesting to speculate on alternative regulatory functions, and on whether these sequences are also conserved in other Drosophilid species.

Exploring the molecular mechanism underlying the cooperative inhibition of $m s l-2$ translation by Sxl and the uORF, the authors [5] found that Sxl binding to the 5' UTR has a two-fold action: it promotes PIC recognition of the uAUG and then freezes or slows its scanning action (Figure 1). Both activities inhibit translation of the downstream major coding sequence. Thus, Sxl acts as a bait to attract the ribosome to the uAUG initiation codon within the UORF, as well as a trap to prevent it from escaping. To investigate the specificity of the RNA-binding protein, the authors [5] replaced Sxl and its binding site with a distinct RNA-binding protein, polypyrimidine tract-binding protein (PTB), and its cognate poly $(\mathrm{CU})$ binding site. Indeed, PTB strongly repressed reporter mRNA translation, but in a uORF-independent way, suggesting that inhibition by the uORF-Sxl couple is not by a simple steric arrest mechanism. Insertion of the essential elements of the msl-2 5' UTR into the human $\beta$ globin 5' UTR allowed Sxl-mediated translational suppression, clearly demonstrating the heterologous nature of the elements. However, the degree of inhibition was less than that of the comparable msl-2 reporter [5], suggesting fine-tuning by cis features (for example, the upstream msl-2 uORFs), and potentially trans-acting factors, that have not yet been identified.

\section{uORF-mediated regulation of translation by SxI might be widespread}

Bioinformatic analysis showed that about half of the 20,000 annotated 5' UTR sequences in the Drosophila melanogaster transcriptome contain one or more uAUGs [5]. The investigators report the presence of 268 Drosophila mRNAs with elements paralleling those in $m s l-2$ : a uAUG appropriately spaced before a poly $(\mathrm{U})$ stretch. The uORFs of 6 of 12 candidate transcripts examined mediated Sxl-dependent suppression of translation, suggesting a rather promiscuous control mechanism. Intriguingly, ribosomal protein RPS14a is a verified target, a finding reminiscent of the 5' UTR-mediated regulation common to metazoan mRNAs encoding ribosomal proteins. However, an analysis of gene ontology is not provided, and there is no information about the possible coherence of the post-transcriptional regulon specified by the uORF-Sxl couple, or on co-regulation of other transcripts involved in sex determination or development [5].

\section{Perspectives and unanswered questions}

Like most important discoveries, the investigation by Hentze and colleagues [5] raises at least as many interesting questions as it answers. The experiments delineate a narrow temporal and spatial window of operation of Sxl after recognition of the translation initiation codon by the PIC but before elongation. The authors speculate that Sxl might influence the conversion of the PIC from the 
scanning-competent open conformation to the scanningarrested closed conformation that is competent for joining of the $60 \mathrm{~S}$ subunit [9]. However, the precise periinitiation step inhibited by Sxl has not yet been identified, nor has subunit joining to form a translation-competent ribosome been determined in the context of the isolated 5' UTR. Likewise, the specific communication between Sxl and the uORF-bound translation machinery remains unknown. Yeast two-hybrid experiments revealed specific interaction of Sxl with eIF3h and eIF3i PIC subunits, but the authors [5] were unable to obtain evidence for a role of these interactions in stalling or locking the PIC on the uORF. Possibly, the inhibitory activity of Sxl requires corepressors to facilitate the interaction with PIC components, as is the case of the 3' UTR where UNR bridges Sxl and poly(A)-binding protein (PABP) to prevent PIC recruitment to the initiation factor complex (Figure 1). It is unlikely that UNR acts as a 5' co-repressor because it would be expected to bind PABP and prevent PIC recruitment (see below). To investigate the possible role of other factors, it will be informative to determine whether recombinant Sxl represses translation of a reporter mRNA containing the msl-2 5' UTR in rabbit reticulocyte or wheat germ lysates in which Drosophila-specific cofactors are likely to be absent.

Previous studies showed that Sxl binding to a poly(U) element in the msl-2 3' UTR blocks initiation by preventing PIC recruitment to the transcript. The authors [5] suggest the dual inhibitory mechanisms might act as a fail-safe system to ensure appropriate $m s l-2$ repression and dosage compensation [7]. To minimize confounding by the 3' UTR-driven mechanism, the authors here [5] have investigated the $m s l-25^{\prime}$ UTR in isolation. Thus, the relative importance of the two mechanisms remains unclear. If the proximal 5'-driven mechanism is highly efficient, then the failure of PIC recruitment would alleviate the necessity for the 3'-driven mechanism. Possibly, both mechanisms are highly efficient, as would be required to silence gene expression completely. An advantage of most translational control mechanisms is their reversibility. However, in this case reversible binding of Sxl to msl-2 UTRs could be detrimental to the organism and leads one to ask why this mode of regulation was selected for this critical developmental process instead of an irreversible, transcription-driven mechanism.
The finding that Sxl co-regulates other Drosophila transcripts in a uORF-dependent manner [5] clearly indicates the global importance of the mechanism, most likely extending beyond sex determination. Importantly, these results introduce the possibility that distinct uORFs cooperate with 5' UTR elements that bind non-Sxl mRNA-binding proteins to regulate translation of other post-transcriptional regulons, and not just in Drosophila, but also in other organisms, including mammals. Finally, these results expand an emerging paradigm in which binary (and possibly ternary) regulatory modules, consisting of nearby mRNA elements and their RNA-binding proteins, behave in a combinatorial, context-specific way to regulate translation and gene expression [10].

Published: 25 July 2011

\section{References}

1. Morris DR, Geballe AP: Upstream open reading frames as regulators of mRNA translation. Mol Cell Biol 2000, 20:8635-8642.

2. Calvo SE, Pagliarini DJ, Mootha VK: Upstream open reading frames cause widespread reduction of protein expression and are polymorphic among humans. Proc Natl Acad Sci U S A 2009, 106:7507-7512.

3. Hinnebusch AG: Translational regulation of GCN4 and the general amino acid control of yeast. Annu Rev Microbio/ 2005, 59:407-450.

4. Yaman I, Fernandez J, Liu H, Caprara M, Komar AA, Koromilas AE, Zhou L, Snider MD, Scheuner D, Kaufman RJ, Hatzoglou M: The zipper model of translational control: a small upstream ORF is the switch that controls structural remodeling of an mRNA leader. Cell 2003, 113:519-531.

5. Medenbach J, Seiler M, Hentze MW: Translational control via proteinregulated upstream open reading frames. Cell 2011, 145:902-913.

6. Kelley RL, Solovyeva I, Lyman LM, Richman R, Solovyev V, Kuroda MI: Expression of msl-2 causes assembly of dosage compensation regulators on the X chromosomes and female lethality in Drosophila. Cell 1995, 81:867-877.

7. Beckmann K, Grskovic M, Gebauer F, Hentze MW: A dual inhibitory mechanism restricts $m s /-2$ mRNA translation for dosage compensation in Drosophila. Cell 2005, 122:529-540.

8. Duncan KE, Strein C, Hentze MW: The SXL-UNR corepressor complex uses a PABP-mediated mechanism to inhibit ribosome recruitment to $\mathrm{msl}-2$ mRNA. Mol Cell 2009, 36:571-582.

9. Passmore LA, Schmeing TM, Maag D, Applefield DJ, Acker MG, Algire MA Lorsch JR, Ramakrishnan V: The eukaryotic translation initiation factors elF1 and elF1A induce an open conformation of the $40 \mathrm{~S}$ ribosome. Mol Cell 2007, 26:41-50.

10. Ray PS, Jia J, Yao P, Majumder M, Hatzoglou M, Fox PL: A stress-responsive RNA switch regulates VEGFA expression. Nature 2009, 457:915-919.

doi:10.1186/gb-2011-12-7-121

Cite this article as: Yao P, Fox PL: Sex lethal and upstream ORFs: a bait-andtrap system for ribosomes. Genome Biology 2011, 12:121. 